\title{
Endovascular Embolization of Intracranial Infectious Aneurysms in Patients Undergoing Open Heart Surgery Using n-Butyl Cyanoacrylate
}

\author{
Esteban Cheng-Ching Seby John Mark Bain Gabor Toth Thomas Masaryk \\ Ferdinand Hui Muhammad Shazam Hussain \\ Cerebrovascular Center of the Neurological Institute, Cleveland Clinic, Cleveland, $\mathrm{OH}, \mathrm{USA}$
}

\begin{abstract}
Keywords
Endovascular embolization · Intracranial infectious aneurysm · Open heart surgery $\cdot \mathrm{n}$-Butyl cyanoacrylate
\end{abstract}

\begin{abstract}
Introduction: Mycotic aneurysms are a serious complication of infective endocarditis with increased risk of intracranial hemorrhage. Patients undergoing open heart surgery for valve repair or replacement are exposed to anticoagulants, increasing the risk of aneurysm bleeding. These patients may require endovascular or surgical aneurysm treatment prior to heart surgery, but data on this approach are scarce. Methods: Retrospective review of consecutive patients with infectious endocarditis and mycotic aneurysms treated endovascularly with Trufill n-butyl cyanoacrylate (n-BCA) at the Cleveland Clinic between January 2013 and December 2015. Results: Nine patients underwent endovascular treatment of mycotic aneurysms with $\mathrm{n}$-BCA (mean age of 39 years). On imaging, 4 patients had intracerebral hemorrhage, 2 had multiple embolic infarcts, and the rest had no imaging findings. Twelve mycotic aneurysms were detected ( 3 patients with 2 aneurysms). Seven aneurysms were in the M4 middle cerebral artery segment, 4 in the posterior cerebral artery distribution, and 1 in the callosomarginal branch. n-BCA was diluted in ethiodized oil (1:1 to 1:2). Embolization was achieved in a single rapid injection with immediate microcatheter removal. Complete aneurysm exclusion was achieved in all cases without complications. All patients underwent open heart surgery and endovascular embolization within a short interval, 2 with both procedures on the same day. There were no new hemorrhages after aneurysm embolization. Conclusions: Endovascular embolization of infectious intracranial aneurysms with liquid embolics can be performed successfully in critically ill patients requiring immediate open heart surgery and anticoagulation. Early embolization prior to and within a short interval from open heart surgery is feasible.
\end{abstract}




\section{Introduction}

Infectious intracranial aneurysms are a rare but serious complication of infective endocarditis. These lesions are typically located at bifurcation points or involving the parent arteries in the distal intracranial circulation, and have been described as thin-walled and fragile, with high predisposition to rupture [1].

At the present time, there are no treatment guidelines for infectious intracranial aneurysms. Antibiotic therapy has been used, with several case series and expert reports suggesting the need for surgery or endovascular embolization in some cases, especially in patients with ruptured aneurysms. However, there is no evidence to determine which the best treatment strategy is. Patients with valve endocarditis undergoing open heart surgery for valve repair or replacement are exposed to large doses of intravenous heparin during surgery, which may increase the risk of mycotic aneurysm bleeding. Often there is reluctance to proceed with open heart surgery due to this risk, and thus, aneurysm treatment in these patients may be indicated, though data on the treatment of unruptured infectious aneurysms as well as the timing in relationship with open heart surgery are scarce [2-5].

We report our experience with endovascular embolization of ruptured and unruptured infectious intracranial aneurysms using the liquid embolic Trufill n-butyl cyanoacrylate (n-BCA) (Codman and Shurtleff Inc., Raynham, MA, USA) in patients undergoing open heart surgery within a short time interval from the embolization procedure.

\section{Methods}

\section{Data Collection}

We retrospectively reviewed a prospectively collected database of neurointerventional procedures performed at the Cleveland Clinic between January 2013 and December 2015. We included consecutive patients with infectious endocarditis and mycotic aneurysms treated endovascularly with the liquid embolic Trufill n-BCA. Data collected included patient demographics, clinical presentation, neuroimaging findings on CT and/or MRI, angiographic findings including aneurysm location and size, and endovascular treatment characteristics. Treatment decision for mycotic aneurysms and clinical outcome on discharge were reviewed.

\section{Patient Assessment Protocol}

At the Cleveland Clinic, all patients with infectious endocarditis requiring open heart surgery or anticoagulation undergo imaging with a non-contrast brain CT scan. If there is any abnormality on the initial imaging, or if the patient has any neurologic symptom, further evaluation is obtained, including neurology consultation and/or further imaging with MRI. Any patient with neurologic symptoms suggestive of central nervous system pathology and/or imaging consistent with ischemic and/or hemorrhagic disease undergoes a diagnostic cerebral angiogram. If an infectious aneurysm is detected, treatment decision is made based on the risk/benefit of aneurysm embolization (including eloquence of the region supplied by the artery involved), rupture status of the aneurysm, and the need for anticoagulation and/or open heart surgery. Individualized decisions are often necessary.

\section{Embolization Technique}

Standard informed consent was obtained prior to all the treatments. The procedures were performed on a biplane angiographic unit (Siemens Artis Q) under general anesthesia, with arterial line blood pressure monitoring. All procedures were performed through a transfemoral arterial access. Intravenous heparin was not used in these cases secondary to the presence of intracerebral hemorrhage and/or hemorrhagic risk of the disease process. After a complete diagnostic angiography, the diagnostic catheter was positioned in the cervical artery (internal carotid or vertebral artery) providing the best access to the intracranial arterial distribution harboring the mycotic aneurysm. The diagnostic catheter was then exchanged for a 6-Fr MPD Envoy guide catheter, which was positioned as distal as feasible in the cervical artery. Under fluoroscopic roadmap guidance, a 165-cm Marathon microcatheter in conjunction with a X-pedion 0.010 microwire or a Mirage 0.008 microwire was navigated to the intracranial artery supplying the mycotic aneurysm, and the 
Table 1. Characteristics of patients with infectious intracranial aneurysms undergoing embolization with Trufill n-butyl cyanoacrylate

\begin{tabular}{|c|c|c|c|c|c|}
\hline $\begin{array}{l}\text { Patient } \\
\text { No. }\end{array}$ & $\begin{array}{l}\text { Imaging } \\
\text { presentation }\end{array}$ & $\begin{array}{l}\text { Infectious aneurysm } \\
\text { location }\end{array}$ & $\begin{array}{l}\text { Aneurysm } \\
\text { size, mm }\end{array}$ & $\begin{array}{l}\text { Reason for } \\
\text { embolization }\end{array}$ & $\begin{array}{l}\text { Timing of cardiac } \\
\text { surgery in } \\
\text { relationship with } \\
\text { embolization }\end{array}$ \\
\hline 1 & Multiple infarcts & Right P3 PCA & $1.4 \times 1.2$ & Presurgery & Same day \\
\hline 2 & $\mathrm{ICH}$ & Left P4 PCA & $3 \times 2.5$ & $\mathrm{ICH}$ & 4 months later \\
\hline 3 & Multiple infarcts & Right M4 MCA & $2.9 \times 2.6$ & Presurgery & 2 days later \\
\hline 4 & ICH & $\begin{array}{l}\text { Right M4 MCA } \\
\text { Right callosomarginal } \\
\text { branch }\end{array}$ & $\begin{array}{c}5 \times 5 \\
1.5 \times 1.4\end{array}$ & $\mathrm{ICH}$ & 5 days prior \\
\hline 5 & $\mathrm{ICH}$ & $\begin{array}{l}\text { Right P4 PCA } \\
\text { Right P4 PCA }\end{array}$ & $\begin{array}{r}1 \times 0.9 \\
1.6 \times 1.2\end{array}$ & ICH & Same day \\
\hline 6 & $\mathrm{ICH}$ & $\begin{array}{l}\text { Right M4 MCA } \\
\text { Left M4 MCA }\end{array}$ & $\begin{array}{l}1.7 \times 1.5 \\
1.8 \times 1.1\end{array}$ & ICH & 4 days later \\
\hline 7 & Negative & Right M4 MCA & $3.8 \times 3.2$ & Presurgery & 7 days later \\
\hline 8 & Negative & Right M4 MCA & $2.3 \times 2.4$ & Presurgery & 5 days later \\
\hline 9 & Negative & Right M4 MCA & $2.5 \times 2.5$ & Presurgery & 3 days later \\
\hline
\end{tabular}

ICH, intracerebral hemorrhage; PCA, posterior cerebral artery; MCA, middle cerebral artery.

tip of the microcatheter was positioned as close as possible to the aneurysm. In a separate sterile table, Trufill $\mathrm{n}$-BCA was prepared and diluted in ethiodized oil in a concentration ranging from 1:1 to 1:2. After flushing the microcatheter with a solution of dextrose $5 \%$ in water, the embolization was performed under high magnification fluoroscopic blank roadmap guidance. The embolization was achieved in a single rapid injection of the embolic material allowing penetration into the aneurysm and including the proximal arterial feeder branch, with subsequent immediate removal of the microcatheter while applying suction with the syringe.

\section{Results}

In the time period included in the study, a total of 9 patients underwent endovascular treatment of intracranial infectious aneurysms using n-BCA (Table 1) (mean age of 39 years, range from 9 to 62 years of age). Eight patients were diagnosed with infectious endocarditis, of whom 6 were found to have vegetations ( 2 in bioprosthetic aortic valves, 1 in a mechanical aortic valve, 1 in a native aortic valve, 2 in native mitral valves), 2 had aortic root abscesses, and $1 \mathrm{had}$ an infected left ventricular assist device. One patient presented with encephalopathy without focal neurologic deficits, and 3 presented with focal neurologic findings. Five patients did not have neurologic symptoms.

Of all the patients, there were 4 with intracerebral hemorrhage and 2 with multiple embolic infarcts on brain imaging. There were no subarachnoid hemorrhages. The reason for cerebral angiography and endovascular embolization of the infectious aneurysms was the presence of intracerebral hemorrhage in 4 patients and preoperative treatment prior to open heart surgery in 5 patients since systemic heparinization was required during these surgeries.

A total of 12 infectious aneurysms were detected ( 3 patients had 2 aneurysms), all in distal branches. Seven infectious aneurysms were in the M4 middle cerebral artery segment, 4 in the distal posterior cerebral artery distribution (1 in the P3 segment and 3 in the P4 


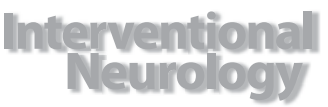

Intervent Neurol 2017;6:82-89

DOI: $10.1159 / 000455806$

Cheng-Ching et al.: Endovascular Embolization of Intracranial Infectious Aneurysms in Patients Undergoing Open Heart Surgery Using n-Butyl Cyanoacrylate
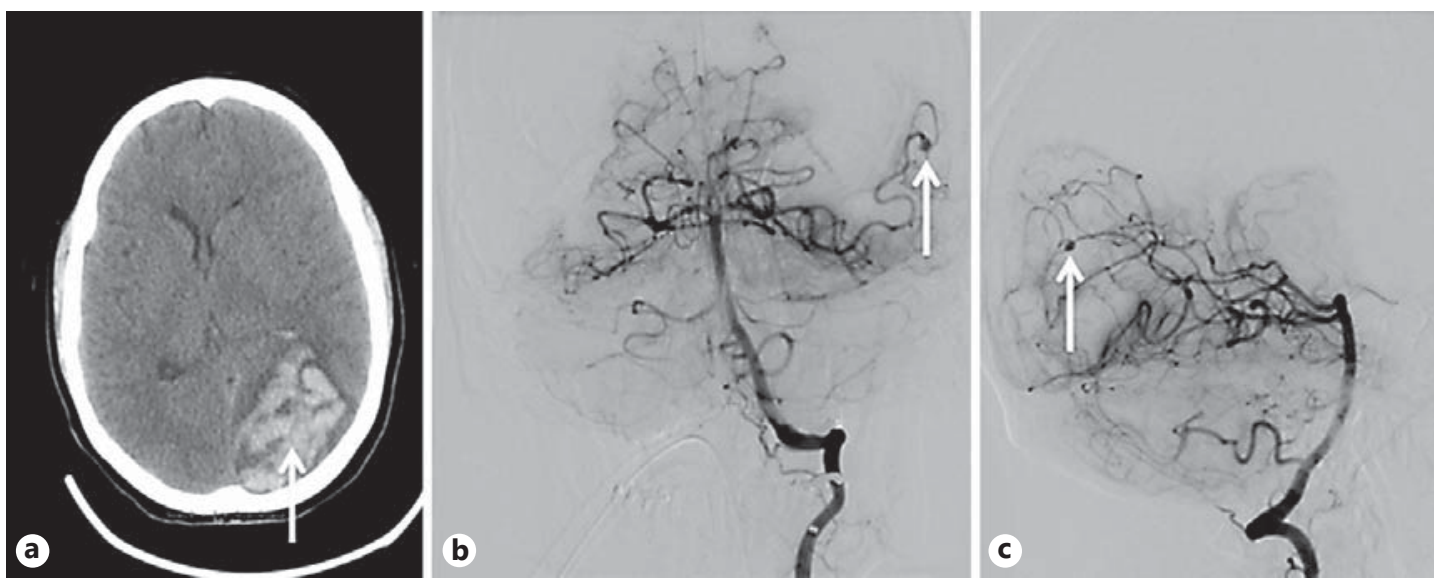

Fig. 1. a Non-contrast axial computed tomography image demonstrates a large left parieto-occipital hemorrhage (arrow) with mass effect and midline shift. Digital subtraction angiography images in posteroanterior (b) and lateral (c) projections demonstrate a mycotic aneurysm in the P4 segment of the left posterior cerebral artery, corresponding to the location of the hemorrhage.

segment), and 1 in the callosomarginal branch. The mean size (greatest diameter) of the infectious aneurysms was $2.4 \mathrm{~mm}$ (range from 0.9 to $5 \mathrm{~mm}$ ).

Embolization of the infectious aneurysms was achieved with complete exclusion of the aneurysm from the circulation in all cases and without periprocedural complications. There was no microcatheter retention nor vessel rupture. All the patients were also treated with antibiotic therapy.

All the patients underwent open heart surgery, 8 undergoing heart valve surgery and 1 requiring heart transplantation. The endovascular embolization occurred prior to the open heart surgery in 8 of the patients, with 2 of these patients having heart surgery the same day of the embolization, and the majority within 7 days of the embolization. One patient had aneurysm embolization 5 days after open heart surgery when he suffered intracerebral hemorrhage secondary to rupture of an infectious aneurysm that was not detected on an angiogram obtained prior to open heart surgery. There was no recurrence of hemorrhage after aneurysm embolization.

On discharge from the hospital, 6 patients had no neurologic deficits, 1 had right upper extremity weakness, 1 had a homonymous hemianopia and 1 had left hemiparesis and hemineglect. These deficits were present prior to embolization.

Patient follow-up ranged from 2 months to 3 years after the endovascular embolization (4 patients had the last follow-up at 3 years, 1 at 5 months, 1 at 4 months, 2 at 3 months, and 1 at 2 months), with no mortality. There were no new neurologic symptoms on follow-up, with 3 patients having unchanged neurologic deficits and the rest with no neurologic deficits. There were no new intracranial hemorrhages reported on follow-up.

\section{Case Example}

A young adult patient with a history of nonischemic cardiomyo pathy with a left ventricular assist device on anticoagulation presented with acute confusion. An emergent head CT demonstrated a large parieto-occipital intracerebral hemorrhage (Fig. 1a). A CT angiogram of the head did not reveal any vascular etiology for the hemorrhage. Anticoagulation was stopped. The patient underwent a cerebral angiogram demonstrating a small mycotic aneurysm in the left posterior cerebral artery P4 segment, corresponding to the area of the 


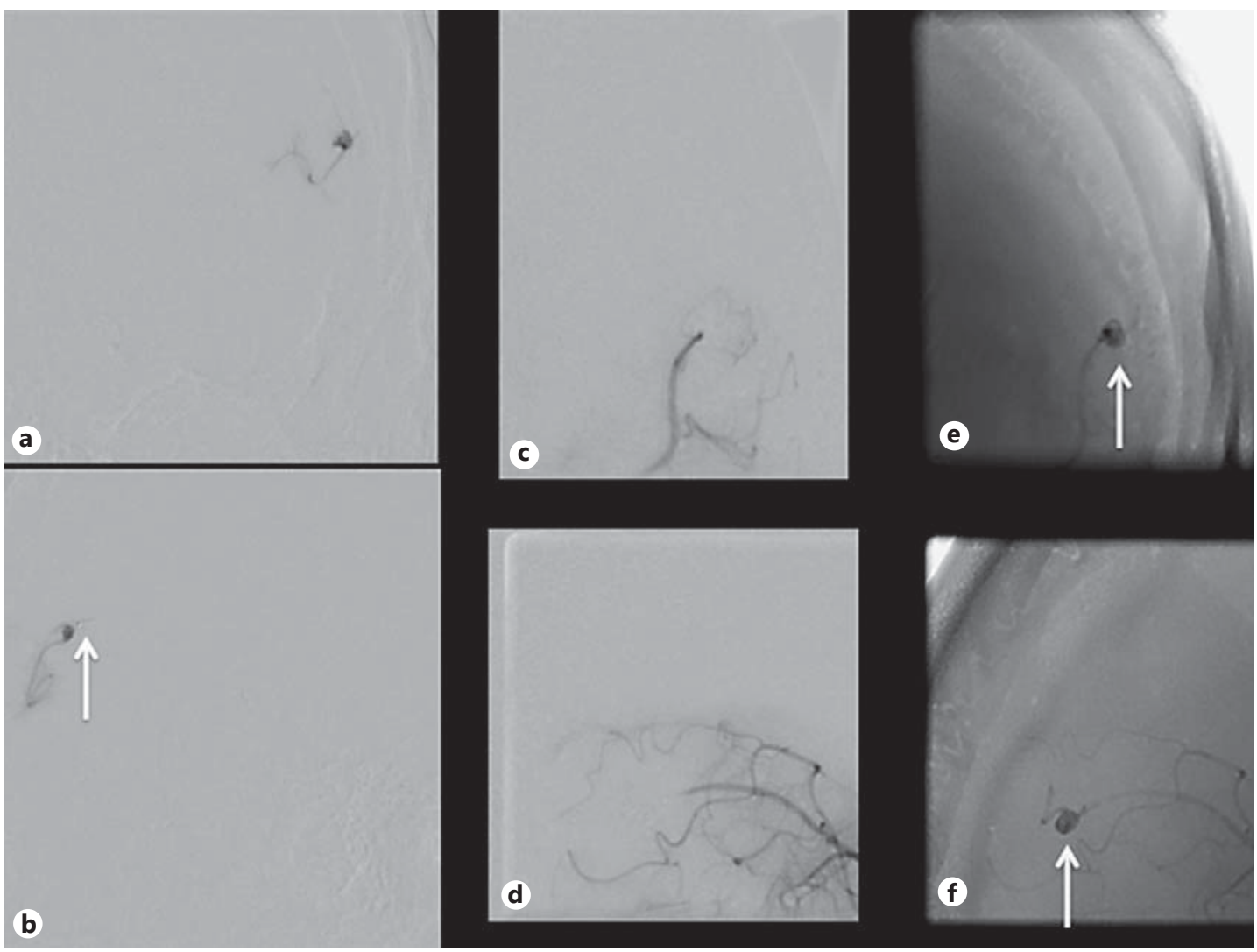

Fig. 2. Digital subtraction angiography images in posteroanterior $(\mathbf{a}, \mathbf{c}, \mathbf{e})$ and lateral $(\mathbf{b}, \mathbf{d}, \mathbf{f})$ projections. Microcatheter injection (a, b) just proximal to the aneurysm prior to embolization (arrow). Magnified view of left vertebral artery injection (c, d) after embolization demonstrates complete occlusion of the aneurysm and the feeding vessel. Corresponding native images $(\mathbf{e}, \mathbf{f})$ demonstrate the n-BCA cast (arrows).

hemorrhage (Fig. 1b, c). The ruptured aneurysm was successfully treated with endovascular Trufill n-BCA embolization, with complete occlusion (Fig. 2). Blood cultures subsequently grew lactobacillus, and the patient was treated with ampicillin, which was continued for a course of 6 weeks with documented clearance of bacteremia. An echocardiogram did not reveal any valvular vegetation, and the bacteremia was considered to be secondary to an infected left ventricular assist device. Anticoagulation was resumed after securing the aneurysm, withoutrecurrence of intracranial hemorrhage. The patient successfully underwent heart surgery. At the 3-year follow-up, the patient is doing well with no recurrence of intracranial hemorrhage.

\section{Discussion}

Infectious aneurysms are a potential and dangerous complication of infectious endocarditis, accounting for $0.7-5.4 \%$ of all intracranial aneurysms [1]. In a series of patients with infectious endocarditis who underwent diagnostic cerebral angiography, $9 \%$ of patients were found to have infectious aneurysms. Presentation with intracranial hemorrhage was a good predictor for aneurysm detection, with $22 \%$ of patients with hemorrhage and infective endocarditis being diagnosed as having an aneurysm in contrast to $1 \%$ when hemorrhage was not present [6]. 
Infectious aneurysms are generally located in distal small branches of the intracranial vasculature. They are often irregular and friable, with potential risk of rupture, leading to subarachnoid and intraparenchymal hemorrhage. Even though the risk of rupture may be low, the mortality rate is high in patients with ruptured infectious aneurysms [2,3]. While antibiotic therapy may be effective in the treatment of some unruptured infectious aneurysms, surgical or endovascular treatments should be considered, especially in those patients with ruptured aneurysms or those at higher risk for intracranial hemorrhage.

Open neurosurgery is a potential treatment option for intracranial infectious aneurysms; however, this may not be feasible in many cases given the frequently encountered complex configuration of the aneurysm, distal location, its relationship with the parent vessel, as well as its fragile structure. In addition, these patients are often critically ill, septic, hemodynamically unstable, and may not tolerate a major open neurosurgical procedure.

Endovascular approach for embolization is evolving and becoming a more frequently used treatment option for infectious intracranial aneurysms. Due to the parent vessel compromise and irregular morphology of these aneurysms, embolization frequently requires vessel sacrifice [7]. This can be accomplished with coils and/or liquid embolics; however, the distal location and small caliber of the parent artery makes coil embolization not feasible in most cases, in whom endovascular embolization with liquid embolics may be the only treatment option.

Liquid embolic agents include ethylene-vinyl alcohol copolymer (Onyx, eV3 Neurovascular, Irvine, CA, USA) and cyanoacrylates. Onyx is a nonadhesive liquid material and its use in the treatment of infectious intracranial aneurysms has been described in the literature [7-9]. Trufill n-BCA is an adhesive, low-viscosity liquid embolic, which polymerizes rapidly. It was approved by the FDA in 2000 for presurgical embolization for arteriovenous malformations $[10,11]$. The use of cyanoacrylates in an exclusive cohort of intracranial infectious aneurysms prior to open heart surgery has not been previously reported. In our series, n-BCA was used exclusively in the treatment of 9 patients with 12 infectious aneurysms, with successful complete obliteration of these lesions. All the cases were performed with a Marathon microcatheter allowing navigation very distally in the intracranial circulation, with rapid injection and penetration into the aneurysm, without any microcatheter retention and without any periprocedural complications. Given the ability to position the microcatheter very close to the infectious aneurysm, rapid polymerization of the liquid embolic was desired. We used a dilution of 1:1 to 1:2 allowing for optimal visualization and relatively rapid polymerization. It is possible that other dilution concentrations may be effective allowing for more distal embolization if optimal catheterization in relationship with the aneurysm cannot be achieved. However, other dilution concentrations may reduce radiopacity and visibility of the liquid embolic.

While embolization of ruptured infectious aneurysms has been widely published, reports on the treatment of unruptured cases are scarce. Data on endovascular embolization prior to open heart surgery are also lacking. Furthermore, there is no available information on the timing of embolization of infectious aneurysms in relationship with the timing of open heart surgery. Patients with infectious intracranial aneurysms undergoing open heart surgery (in whom heparinization is needed) and those requiring systemic anticoagulation are at high risk for intracranial hemorrhage, and treatment of the aneurysm should be strongly considered. Evidence to support this approach in patients with infectious aneurysms undergoing open heart surgery is very limited. Patients who require valve surgery or other type of open heart surgery may be more critically ill and at higher morbidity and mortality risk as compared to those who may not need cardiac surgery, and this may account for the lack of studies including these patients. A recently published paper by Singla et al. [12], performed using the Nationwide Inpatient Sample database on the national treatment practices in the management of infec- 
tious intracranial aneurysms and infective endocarditis, revealed that the majority of patients with infectious intracranial aneurysms were managed conservatively. In this cohort, $18.6 \%$ underwent endovascular embolization or surgical clipping of the aneurysm independently of the rupture status, with only $18 \%$ of ruptured aneurysms and $25 \%$ of unruptured aneurysms undergoing treatment to exclude the aneurysm. The mortality was higher in patients managed nonoperatively (26.7\%) as compared to those who had endovascular embolization or surgical clipping of the aneurysm (15.1\%). In addition, cardiac procedures were only performed in $18.6 \%$ of patients with ruptured aneurysms and $15.6 \%$ of those with unruptured aneurysms. Only $1.5 \%$ of patients had cardiac surgery and aneurysm treatment within the same admission [12].

In our series, all of our patients had intracranial infectious aneurysms and underwent successful open heart surgery, two of them on the same day of the endovascular embolization of the aneurysm, and most of them on the same admission within a short time frame after the embolization. These patients did not have any increased risk of complications, and the endovascular embolization was feasible and effective, preventing hemorrhage during the cardiac surgical procedure in which systemic heparinization was used, and preventing subsequent intracranial bleeding. Our strategy to treat intracranial infectious aneurysms with endovascular embolization regardless of the rupture status, allowed the possibility to perform immediate open heart surgery and systemic anticoagulation with reduced risk of intracranial hemorrhage. Timing of embolization may be important, and this approach with early embolization may contribute to the safety of open heart surgery from the neurologic standpoint by reducing the intracranial bleeding risk, permitting cardiac surgical procedures and anticoagulation in those patients previously considered not candidates, reducing the waiting time for open heart surgery, and therefore possibly increasing the likelihood of survival of patients with infectious intracranial aneurysms and infectious endocarditis and improving their neurologic outcomes. Early infectious aneurysm embolization should be considered in this patient population.

\section{Limitations}

This is a single center study and has significant limitations due to its retrospective nature and small number of patients, which introduces selection bias. A larger multicenter study may be required to confirm the safety and efficacy of this treatment option. Long-term followup is needed to determine durability of this embolization strategy.

\section{Conclusions}

Endovascular embolization of infectious intracranial aneurysms with liquid embolics can be performed successfully in critically ill patients requiring immediate open heart surgery and systemic anticoagulation. This treatment may be indicated for ruptured infectious aneurysms, or in those patients with unruptured intracranial infectious aneurysms requiring anticoagulation or undergoing open heart surgery. Early embolization prior to and within a short interval from open heart surgery is feasible and should be considered in these patients.

\section{Disclosure Statement}

The authors fully participated in the writing of this paper adhering to strict ethical standards. All authors report no conflict of interest or financial disclosures. 
Cheng-Ching et al.: Endovascular Embolization of Intracranial Infectious Aneurysms in Patients Undergoing Open Heart Surgery Using n-Butyl Cyanoacrylate

\section{References}

1 Ducruet AF, Hickman ZL, Zacharia BE, Narula R, Grobelny BT, Gorski J, Connolly ES Jr: Intracranial infectious aneurysms: a comprehensive review. Neurosurg Rev 2010;33:37-46.

2 Kannoth S, Iyer R, Thomas SV, Furtado SV, Rajesh BJ, Kesavadas C, Radhakrishnan VV, Sarma PS: Intracranial infectious aneurysm: presentation, management and outcome. J Neurol Sci 2007;256:3-9.

3 Kannoth S, Thomas SV: Intracranial microbial aneurysm (infectious aneurysm): current options for diagnosis and management. Neurocrit Care 2009;11:120-129.

4 Venkatesh SK, Phadke RV, Kalode RR, Kumar S, Jain VK: Intracranial infective aneurysms presenting with haemorrhage: an analysis of angiographic findings, management and outcome. Clin Radiol 2000;55:946-953.

5 Peters PJ, Harrison T, Lennox JL: A dangerous dilemma: management of infectious intracranial aneurysms complicating endocarditis. Lancet Infect Dis 2006;6:742-748.

6 Hui FK, Bain M, Obuchowski NA, Gordon S, Spiotta AM, Moskowitz S, Toth G, Hussain S: Mycotic aneurysm detection rates with cerebral angiography in patients with infective endocarditis. J Neurointerv Surg 2015;7: 449-452.

7 Grandhi R, Zwagerman NT, Linares G, Monaco EA 3rd, Jovin T, Horowitz M, Jankowitz BT: Onyx embolization of infectious intracranial aneurysms. J Neurointerv Surg 2014;6:353-356.

8 Jadhav AP, Pryor J, Nogueira RG: Onyx embolization for the endovascular treatment of infectious and traumatic aneurysms involving the cranial and cerebral vasculature. J Neurointerv Surg 2013;5:562-565.

9 Chalouhi N, Tjoumakaris S, Gonzalez LF, Hasan D, Alkhalili K, Dumont AS, Rosenwasser R, Jabbour P: Endovascular treatment of distal intracranial aneurysms with Onyx 18/34. Clin Neurol Neurosurg 2013;115:25282532.

10 Rosen RJ, Contractor S: The use of cyanoacrylate adhesives in the management of congenital vascular malformations. Semin Intervent Radiol 2004;21:59-66.

11 Elsenousi A, Aletich VA, Alaraj A: Neurological outcomes and cure rates of embolization of brain arteriovenous malformations with n-butyl cyanoacrylate or Onyx: a metaanalysis. J Neurointerv Surg 2016;8:265-272.

12 Singla A, Fargen K, Blackburn S, Neal D, Martin TD, Hess PJ, Beaver TM, Klodell CT, Hoh B: National treatment practices in the management of infectious intracranial aneurysms and infective endocarditis. J Neurointerv Surg 2016;8:741-746. 